\title{
Benign Glomus Tumor
}

National Cancer Institute

\section{Source}

National Cancer Institute. Benign Glomus Tumor. NCI Thesaurus. Code C6748.

A glomus tumor of small size characterized by the absence of nuclear atypia and mitotic activity. 